Background The laboratory diagnosis of syphilis is a crucial point in the diagnostic evaluation of the syphilis. The aim of this study was to evaluate the diagnostic performance of the new commercial immunoblotting "recomLine Treponema" (Mikrogen Diagnostik, Germany) assay which includes two new recombinant antigens (Tp257 (Gpd) and Tp453) in addition to the four (Tp47, TmpA, Tp17, Tp15) shown in a previous version.

Methods The presence of specific antibody response to T. pallidum was evaluated by comparing the immunoblotting test, that detects both IgG and IgM antibodies, with the "Syphilis TP" (Architect system, Germany) immunoassay, that detects the total T. pallidum specific antibody and routinely used in our Hospital for serological screening. The serum samples included in this study were obtained from 112 patients with suspect or clinical evidence of syphilis infection.

Results Of the 112 samples analysed for specific T. pallidum antibody by "Syphilis TP" assay, 90 samples were detected positive, 8 negative, 10 borderline and 4 samples showed an not interpretable result.

Of the 112 samples analysed for specific IgG and IgM antibodies by "recomLine Treponema" assay, 97 (86.61\%) specimens resulted positive (100\% were IgG positive and $16.49 \%$ were also IgM positive), $7(6.25 \%)$ negative and $8(7.14 \%)$ borderline (6 for the $\operatorname{IgG}$ and 2 for the IgM).

Conclusion The comparison between the two test showed that the "recomLine Treponema" assay identified more positive sample, less negative and borderline samples and not interpretable results.

This preliminary results underline that the "recomLine Treponema" (Mikrogen Diagnostik, Germany) test is clinically valid not only because it can be used as confirmatory test but also because it allows to discriminate between IgG and IgM antibodies.

Future objectives of this study will be to validate the "recomLine Treponema" test on other groups of subjects and compare it with other serological tests.

\section{P2.066 COMPARING THE PERFORMANCE CHARACTERISTICS OF CSF-TRUST AND CSF-VDRL FOR SYPHILIS: A CROSS-SECTIONAL STUDY}

doi:10.1136/sextrans-2013-051184.0331

'W Gu, 'Y Yang, 'L Wu, 'S Yang, 'L Ng. 'Shanghai Skin Disease Hospital, Shanghai, China; ${ }^{2}$ National Microbiology Laboratory, Public Health Agency of Canada, Winnipeg, MB, Canada

Background In the past decade, China has observed an annual increasing rate of syphilis over $14 \%$ and the cases of neurosyphilis were increased accordingly. The WHO standard method for screening neurosyphilis is examination of CSF fluid and a mandatory VDRL laboratory procedure. The situation is even more challenging in China as there are no commercial VDRL reagents approved by the SFDA for CSF-VDRL examination. In this study we aimed to determine the performance characteristics of CSF-TRUST as compared to CSF-VDRL for laboratory diagnosis of neurosyphilis.

Methods CSF and serum samples were collected from 824 individual STD clinic patients who have syphilis and are suspected to progress to neurosyphilis in a 9 -month period. CSF-VDRL and CSFTRUST were performed in parallel on the same day when collected. TPPA tests were also performed on the CSF and the serum samples, and Biochemical analysis of the CSF samples was also performed.

Results The overall agreement between CSF-TRUST and CSFVDRL was $97.3 \%$. The reactive ratios of the CSF samples were $22.1 \%$ by CSF-TRUST and $24.8 \%$ by CSF-VDRL, respectively. All CSF-TRUST reactive cases were reactive in the CSF-VDRL. Twentytwo samples with CSF-TRUST negative were tested CSF-VDRL reactive with low titers (1:1 to $1: 4)$. Over $97 \%$ of the double reactive CSF samples (CSF-VDRL and CSF-TRUST) had an identical titer or a titer within a 2 -fold difference. The agreement of CSF-TPPA and
CSF-VDRL was $71.9 \%$. Similarly the agreement of CSF-TPPA and CSF-TRUST was $69.2 \%$.

Conclusions TRUST reagent kit is commercially available in China with SFDA certificate. It seems that CSF-TRUST and CSFVDRL are congruence in most cases, but CSF-TRUST is less sensitive than CSF-VDRL. Nevertheless, our results suggested that CSF-TRUST may be used as an alternative for laboratory diagnosis of neurosyphilis in clinical settings with CSF-VDRL unavailable.

\section{P2.067 SYPHILIS TESTING IN THE PUBLIC HEALTH SETTING IN NORTH RHINE-WESTPHALIA, 2011-2012}

doi:10.1136/sextrans-2013-051184.0332

'A Lucht, ${ }^{2}$ S Kuttner-May, 'D Münstermann, 'H J Hagedorn. 'Labor Krone GbR, Bad Salzuflen, Germany; ${ }^{2}$ Landeszentrum Gesundheit NRW, Münster, Germany

Background According to the Robert-Koch-Institut (RKI) syphilis incidence is increasing in Germany especially among men who have sex with men (MSM). North Rhine-Westphalia (NRW) is the federal state with the highest number of syphilis infections. By order of the Ministry of Health the Landeszentrum Gesundheit (lzg.nrw) organises and supports anonymous syphilis testing by local public health authorities (LPHA). Aim of this study was to assess how many syphilis cases could be detected and if hard-to-reach risk groups use the possibility of syphilis testing in this public health setting.

Methods 46 out of 53 LPHA in NRW offered their clients after counselling a syphilis screening test (chemiluminiscent microparticle immunoassay, CMIA, Abbott) performed in the German syphilis consiliary laboratory (Labor Krone, Bad Salzuflen). Reactive tests were confirmed by treponema pallidum particle agglutination assay (TPPA) and fluorescent treponemal antibody absorption test (FTA-abs)-IgG and further tested by 19s-IgM-FTA-abs and rapid plasma reagin assay (RPR). Results In 2011-2012, 7961 clients were tested. There were 54.8\% men, $44.4 \%$ women, mean age was 32.8 years, 35.8\% were MSM, $28.4 \%$ female sex workers (FSW). 705 reactive tests could be confirmed by further analysis. $17.2 \%$ positive results were classified as active infections, $7.5 \%$ as suspected latent infections, and $75.3 \%$ as known, already treated treponemal infections. In 2011, the LPHA detected 79 out of 986 notifiable syphilis infections in NRW with higher proportions of MSM (67.1\%) and women (22.7\%), most of them FSW, in comparison to $52.7 \%$ MSM and $9.1 \%$ women in NRW reported to RKI.

Discussion Approximately $87 \%$ of the LPHA in NRW offered syphilis testing. A relevant number of active and latent syphilis was detected among LPHA clients and it could be shown that the LPHA were able to detect syphilis especially in high risk groups such as MSM and FSW which might have otherwise not been tested.

\section{P2.068 STUDY ON THE PERFORMANCE OF THE DETERMINE ${ }^{\circledR}$ SYPHILIS RAPID TEST}

doi:10.1136/sextrans-2013-051184.0333

${ }^{1}$ H J Hagedorn, ${ }^{2} \mathrm{~A}$ Kraminer-Hagedorn, ${ }^{3} \mathrm{~S}$ Kuttner-May, ${ }^{4} \mathrm{H}$ Nitschke, ${ }^{1} \mathrm{~A}$ Lucht, 'D Münstermann, ${ }^{5} \mathrm{~N}$ H Brockmeyer. 'Labor Krone GbR, Bad Salzuflen, Germany; ${ }^{2}$ Spirolab GmbH, Bad Salzuflen, Germany; 'andeszentrum Gesundheit Nordrhein-Westfalen, Muenster, Germany; ${ }^{4}$ Gesundheitsamt, Koeln, Germany; ${ }^{5}$ Klinik für Dermatologie und Venerologie der Ruhruniversität, Deutsche STI Gesellschaft, Bochum, Germany

Background The usefulness of syphilis rapid tests in STI counselling services is under discussion in Germany. For this reason, we evaluated the performance of the Determine ${ }^{\circledR}$ Syphilis rapid test (TP-RT) in comparison to standard serological syphilis tests.

Materials and Methods The TP-RT was carried out in 2,203 serum or plasma samples from the German syphilis consiliary laboratory (Labor Krone GbR, Bad Salzuflen) representing a broad spectrum of relevant samples from public health institutions, STI and HIV ambulances, hospitals and others, including samples from 532 MSM, 417 female sex workers and 260 pregnant women. The 